\title{
FINITE DIMENSIONAL SUBALGEBRAS IN MATRIX RINGS OVER TRANSCENDENTAL DIVISION ALGEBRAS
}

\author{
A. I. LICHTMAN AND B. A. F. WEHRFRITZ
}

(Communicated by D. S. Passman)

\begin{abstract}
We prove in this article that for some classes of division algebras $D$ over a field $F$ every finite dimensional semisimple subalgebra of $D^{n \times n}$ must be conjugate to a subalgebra of $F^{n \times n}$.
\end{abstract}

Quite a body of information has been amassed in recent years about groups of matrices over various examples of division algebras of a vaguely transcendental nature. Our object here is to provide a general framework sufficient to derive the properties of at least the locally finite such groups.

Throughout this paper $F$ is a (commutative) field, $D$ is a division $F$-algebra and $n$ is a positive integer. There are various notions of transcendence for division algebras. We shall be concerned here with the following, or weakened versions of it: $K \otimes_{F} D$ is a domain for every finite-dimensional division $F$ algebra $K$. Such division algebras are called totally transcendental in the book [15]. Note that $K \otimes_{F} D$ has finite dimension $\operatorname{dim}_{F} K$ over $D$ as left or right space; in particular $K \otimes_{F} D$ is a domain if and and only if it is a division ring.

The following examples of division algebra do have the above property.

(a) The division ring $D$ of quotients of the universal enveloping algebra $U(L)$ of a Lie F-algebra $L$ such that $U(L)$ is Ore.

For example $L$ could be any soluble by finite-dimensional Lie $F$-algebra, see [11, Proposition 4.1] or [12, Theorem 1, Corollary]. More generally $L$ could be any Lie algebra in the class $\langle L, P\rangle \mathfrak{F}_{F}$ generated by the local and extension closure operators $L$ and $P$ and the class $\mathfrak{F}_{F}$ of finite-dimensional Lie $F$ algebras.

(b) The division ring $D$ constructed by $P . M$. Cohn in [1] from $U(L)$ for $L$ any Lie F-algebra.

(c) The division ring $D$ of quotients of a group algebra $F G$ for any group $G$ for which $K G$ is Ore for all such $K$.

Received by the editors June 10, 1988 and, in revised form, October 5, 1988.

1980 Mathematics Subject Classification (1985 Revision). Primary 16A40, 16A46; Secondary $20 \mathrm{H} 25$.

The first author was supported by NSF Grant DMS 8802634. 
Here $G$ could be any torsion-free locally soluble-by-finite group, or more generally any group in $\langle L, P\rangle(\mathfrak{A F})$, see [8, Theorem 1.4].

(d) The Malcev-Neumann power series ring $D=F\langle G\rangle$ constructed for any ordered group $G$ and hence for any residually torsion-free nilpotent group $G$.

In particular, via the results of [9], the ring $D$ could be the universal field of fractions of a free $F$-algebra.

Our group theoretic results apply to locally finite groups. We remark here, and once and for all, that in certain cases they extend to periodic groups. Specifically in the following cases the periodic subgroups of $\operatorname{GL}(n, D)$ are known to be locally finite: $D$ as in (a) with $L$ either locally finite-dimensional, or metabelian, or residually nilpotent, see $[11$, p. 34,35$], D$ as in (c) with $G$ polycyclic-byfinite ([12]) or with $G$ in certain more general classes of group ([11, Part II] and $[16,4.48 \mathrm{f}])$, and $D$ as in (d) with $G$ residually torsion-free nilpotent, see [5].

We now state our main results. The positive group theoretic content is in the first theorem below. We remind the reader that $D$ is a division algebra over the field $F$. Also $D^{n \times n}$ denotes the ring of $n \times n$ matrices over $D$.

1. Theorem. Assume $K \otimes_{F} D$ is a domain for every finite-dimensional separable division $F$-algebra $K$.

(a) Suppose $R$ is a finite-dimensional separable $F$-subalgebra of $D^{n \times n}$. Then $\operatorname{dim}_{F} R \leq n^{2}$ and $u^{-1} R u \subseteq F^{n \times n}$ for some $u \in \mathrm{GL}(n, D)$. Further $\operatorname{dim}_{F} R$ divides $n^{2}$ if $R$ is simple, divides $n$ if $R$ is a division ring and is at most $n$ if $R$ is commutative.

(b) Let $G$ be a locally finite subgroup of $\mathrm{GL}(n, D)$ with unipotent radical $u(G)$. Then $G / u(G)$ is isomorphic to a subgroup of $\mathrm{GL}(n, F)$ and if further $G$ has no non-trivial elements of order char $F$ then $u^{-1} G u \subseteq \mathrm{GL}(n, F)$ for some $u \in \mathrm{GL}(n, D)$.

Thus the study of locally finite subgroups of $\operatorname{GL}(n, D)$ is reduced essentially to a study of linear groups over the specified field $F$. Most earlier results of this type have constructed representations either over the complex numbers or over the algebraic closure of a finite field (e.g. $[16,2.3 .1,2.5 .1,2.5 .2,2.5 .3$, 2.5.6 and 4.4.9]). Note that in 1. the field $F$ can, and usually will, be very much smaller than the centre of $D$.

We have assumed in 1 . that $D$ has an apparently weaker property than that discussed above, since that is all that is needed to cope with the group-theoretic situation. Under the full hypothesis one can slightly strengthen the ring theoretic conclusions.

2. Theorem. Assume $K \otimes_{F} D$ is a domain for every finite-dimensional division $F$-algebra $K$. Suppose $R$ is a finite-dimensional semisimple $F$-subalgebra of $D^{n \times n}$. Then $\operatorname{dim}_{F} R \leq n^{2}$ and $u^{-1} R u \leq F^{n \times n}$ for some $u \in \operatorname{GL}(n, D)$. Further $\operatorname{dim}_{F} R$ divides $n^{2}$ if $R$ is simple, divides $n$ if $R$ is a division ring and is at most $n$ if $R$ is commutative. 
It is very hard to characterize even the commutative division rings $D$ as in 2., or for that matter as in 1. In some ways it is more natural to consider division $F$-algebras $D$ with the weaker property that $K \otimes_{F} D$ is a domain for every finite field extension $K$ of $F$. A fact we make no use of is that a division $F$-algebra $D$ has this property if and only if $K \otimes_{F} D$ is an Ore domain for every field extension $K$ of $F$, see [3, Corollary 6]. Under this weaker hypothesis part but not all of the conclusion of 1 . and 2 . survive.

3. Theorem. Assume $K \otimes_{F} D$ is a domain for every finite separable field extension $K$ of $F$.

(a) Suppose $R$ is a finite-dimensional separable $F$-subalgebra of $D^{n \times n}$. Then $\operatorname{dim}_{F} R \leq n^{2}$. If $R$ is simple then $\operatorname{dim}_{F} R$ divides $n^{2}$. If $R$ is a field then $\operatorname{dim}_{F} R$ divides $n$. If $R$ is commutative then $\operatorname{dim}_{F} R \leq n$ and $u^{-1} R u \subseteq$ $F^{n \times n}$ for some $u \in \mathrm{GL}(n, D)$. There exist examples with $R$ a division ring, $\operatorname{dim}_{F} R>n$ and with no isomorphic copy of $R$ lying in $F^{n \times n}$. Moreover for finite-dimensional simple inseparable $F$-subalgebras $S$ of $D^{n \times n}$ there need be no bound on $\operatorname{dim}_{F} S$, even if $S$ is 1-generator and $n=1$.

(b) Suppose $G$ is a locally finite subgroup of $\operatorname{GL}(n, D)$ and denote by $Q=$ $F[G]$ the $F$-subalgebra of $D^{n \times n}$ generated by $G$. Then $Q=R_{0} \oplus \mathfrak{n}(Q)$ for some finite-dimensional separable $F$-subalgebra $R_{0}$ of $Q$ and $\mathfrak{n}(Q)$ the nilpotent radical of $Q$. There exist examples of such groups $G$ that are finite with trivial unipotent radical and not isomorphic to any subgroup of $\mathrm{GL}(n, F)$.

Note that any nil subring of $D^{n \times n}$ is nilpotent by Levitzki's theorem, so any subring $S$ of $D^{n \times n}$ does have a nilpotent radical $\mathfrak{n}(S)$.

4. Theorem. Assume $K \otimes_{F} D$ is a domain for every finite field extension $K$ of $F$. Suppose $R$ is a d-generator finite-dimensional $F$-subalgebra of $D^{n \times n}$. Then

$$
\begin{aligned}
\operatorname{dim}_{F} R & \leq n^{2}\left(1+d n^{2}+\cdots+\left(d n^{2}\right)^{n-1}\right) \text { in general and } \\
& \leq n\left(1+\left(\begin{array}{c}
d \\
1
\end{array}\right)+\cdots+\left(\begin{array}{c}
d+n-2 \\
n-1
\end{array}\right)\right) \text { if } R \text { is commutative. }
\end{aligned}
$$

Further $\operatorname{dim}_{F} R$ is at most $n^{2}$ if $R$ is semisimple, divides $n^{2}$ if $R$ is simple and divides $n$ if $R$ is a field. Also if $R$ is commutative and semisimple then $\operatorname{dim}_{F} R \leq n$ and $u^{-1} R u \leq F^{n \times n}$ for some $u \in \mathrm{GL}(n, D)$. There exist examples with $R$ a division ring, $\operatorname{dim}_{F} R>n$ and with no isomorphic copy of $R$ lying in $F^{n \times n}$.

In general in 4. there is no bound on $\operatorname{dim}_{F} R$ independent of $d$, even if $R$ is commutative. More generally if $D$ is as in 2 . with $F \neq D$, then $\operatorname{dim}_{F} D$ is infinite and

$$
\left\{\left(\begin{array}{ll}
a & 0 \\
b & a
\end{array}\right): a \in F, b \in D\right\}
$$

is a locally finite-dimensional commutative $F$-algebra of infinite dimension. 
We make no attempt to obtain the best bounds in 4 ., but the given bounds are not wildly large. For suppose $x_{1}, x_{2}, \ldots, x_{d}$ are non-commuting indeterminates over a field $F$. Then $F\left[x_{1}, \ldots, x_{d}\right]$ lies in a division $F$-algebra $D$ satisfying the hypotheses of 4 . (indeed satisfying the hypotheses of 2.). Suppose $R$ is the $F$-subalgebra of $D^{n \times n}$ generated by the elements

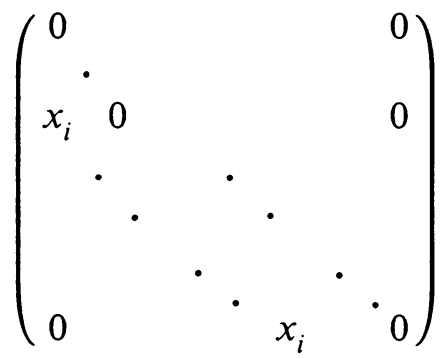

for $i=1,2, \ldots, d$. It is easy to check that $\operatorname{dim}_{F} R=1+d+\cdots+d^{n-1}$. Trivially $\operatorname{dim}_{F} F^{n \times n}=n^{2}$. If the $x_{i}$ are now taken to be commuting indeterminates then $D$ still exists and $\operatorname{dim}_{F} R=1+\left(\begin{array}{c}d \\ 1\end{array}\right)+\cdots+\left(\begin{array}{c}d+n-2 \\ n-1\end{array}\right)$. Of course the full diagonal algebra in $F^{n \times n}$ is commutative of dimensional $n$.

Since the examples we construct for $G$ in (3b) have characteristic zero, the whole of $(3 b)$ remains true under the hypothesis of 4 . We conclude this discussion of the conclusions of the theorems with the following simple corollary.

5. Corollary. Let $D$ be as in 4. An algebraic F-subalgebra $A$ of $D^{n \times n}$ is a $P I$-algebra and in particular is locally finite-dimensional over $F$.

For if $a \in A$ in 5 . the $F$-subalgebra of $D^{n \times n}$ generated by $a$ has dimension at most $n^{2}$ over $F$ (take $d=1$ in 4 . and note that the subalgebra is commutative). Thus $A$ is a $P I$-algebra by [6, Theorem 1 of Section X.10]. Consequently $A$ is also locally finite-dimensional over $F$, see [6, Section X.12, Theorem 1].

We should discuss the extent to which the various hypotheses above actually differ. Fairly standard field theory, e.g. see [7, especially Section IV.10], yields the following.

6. Let $E$ be a field extension of the field $F$.

(a) $K \otimes_{F} E$ is a domain for every separable field extension $K$ of $F$ if and only if it is a domain for every finite separable field extension $K$ of $F$, if and only if $F$ is separably algebraically closed in $E$.

(b) $K \otimes_{F} E$ is a domain for every field extension $K$ of $F$ if and only if it is a domain for every finite field extension $K$ of $F$, if and only if $F$ is algebraically closed in $E$ and $E$ is separable over $F$.

Thus for example, if $E$ is any non-trivial purely inseparable extension field of $F$ then $E$ satisfies the conditions of (6a) but not those of (6b).

Let $F$ be a field of characteristic not 2 with elements $a$ and $b$ such that the quaternion algebra $A=(a, b / F)$ is a division ring. (For the definition 
of $A$ see for example [14, p. 14]). For example we could choose $F$ to be any subfield of $\mathbf{R}$ and $a=b=-1$ or we could take $K$ to be any field with char $K \neq 2, a$ and $b$ independent indeterminates over $K$ and $F=K(a, b)$, see [14, p. 15]. If $X$ and $Y$ are independent indeterminates over $F$ then a simple direct argument shows that $a X^{2}+b Y^{2}-1$ is irreducible in $F[X, Y]$. Thus $F[X, Y] /\left(a X^{2}+b Y^{2}-1\right)$ is a domain; let $E$ denote its quotient field. Then $(E: F(X))=2$, where $F(X)$ is purely transcendental over $F$ and $E$ is separable over $F$. A direct calculation shows that $F$ is algebraically closed in $E$. By hypothesis $A$ is a central division algebra of index 2 and it follows from $[14$, p. 15 , Proposition], that

$$
A \bigotimes_{F} E=(a, b / E) \cong E^{2 \times 2}
$$

In particular $A \otimes_{F} E$ is not a domain. This and 6. yield Part (a) of the following.

7. (a) For the fields $E \supseteq F$ described above $K \otimes_{F} E$ is a domain for every field extension $K$ of $F$ but not for every finite-dimensional separable division $F$-algebra $K$.

(b) For every characteristic $p \geq 0$ there is a field extension $E \supseteq F$ of characteristic $p$ such that $K \otimes_{F} E$ is a domain for every separable field extension $K$ of $F$ but not for every finite-dimensional separable division $F$-algebra $K$.

Part (a) of 7. already affords examples for Part (b) whenever $p \neq 2$. We give a second construction giving examples for all $p>0$. Thus let $p>0$. The skew polynomial ring $\operatorname{GF}\left(p^{p}\right)[x]$, where $x$ acts on the coefficient field as the Frobenius automorphism, is an Ore domain; let $B$ be its division ring of quotients. Then $F=\operatorname{GF}(p)\left(x^{p}\right)$ in the centre of $B$ and $\operatorname{dim}_{F} B=p^{2}$. Set $E=\mathrm{GF}(p)(x)$. Then $(E: F)=p$ and $E$ splits $B$. Thus $B \otimes_{F} E \cong E^{p \times p}$ is not a domain. Trivially $B$ is separable over $F$. Finally $E$ is purely inseparable over $F$ and so $K \otimes_{F} E$ is a domain for every separable field extension $K$ of $F$ by (6a).

We are grateful to the referee for pointing out that if $F$ is a purely transcendental extension of transcendence degree 1 over an algebraically closed field of positive characteristic, then every finite-dimensional division $F$-algebra is a field ([14, p. 376, Corollary a]), so if $E$ is a nontrivial purely inseparable extension field of $F$ then $K \otimes_{F} E$ is domain for every finite-dimensional separable division $F$-algebra $K$ by (6a) but not for every finite-dimensional division $F$-algebra $K$ by $(6 \mathrm{~b})$

We now present the main proofs. Basically we regard the conjugacy parts as variants of the Skolem-Noether theorem and copy a standard method for proving the latter result. For the next three results $R$ is a finite-dimensional $F$-subalgebra of the matrix ring $D^{n \times n}$. Further $R^{\text {op }}$ denotes the opposite ring to $R$. 
8. Suppose $R$ is a division algebra and $R^{\mathrm{op}} \otimes_{F} D$ is a domain. Then $r=\operatorname{dim}_{F} R$ divides $n$ and $u^{-1} R u \subseteq F^{n \times n}$ for some $u \in \mathrm{GL}(n, D)$.

Proof. Its natural $R-D^{n \times n}$ bimodule structure makes $D^{n \times n}$ into a right $S=$ $R^{\mathrm{op}} \otimes_{F} D^{n \times n}$ module. By hypothesis $R^{\mathrm{op}} \otimes_{F} D$ is a domain of finite dimension $r$ over $D$. Thus $R^{\mathrm{op}} \otimes_{F} D$ is a division ring and $S \cong\left(R^{\mathrm{op}} \otimes_{F} D\right)^{n \times n}$ is simple Artinian. As such $S$ has up to isomorphism a unique irreducible right module $V \cong\left(R^{\mathrm{op}} \otimes_{F} D\right)^{(n)}$ and $\operatorname{dim}_{D} V=r n$, the $D$-module structure being given via $D \rightarrow 1 \otimes D \leq S$. Also $D^{n \times n} \cong_{S} V^{(s)}$ for some positive integer $s$. Thus $n^{2}=r n s$ and $r$ divides $n$ as claimed.

It follows that $R$ is isomorphic to an $F$-subalgebra, say $R_{1}$, of $F^{n \times n}$, for example via right multiplication by $R$ on $R^{(s)}$. Thus $D^{n \times n}$ can also be made into a right $S$-module via its natural $R_{1}-D^{n \times n}$ bimodule structure and some fixed isomorphism $\theta: R \rightarrow R_{1}$. Dimension considerations show that it too is $S$-isomorphic to $V^{(s)}$. Consequently there is an $F$-isomorphism $\phi$ of $D^{n \times n}$ to itself such that $(t x y) \phi=t \theta x \phi y$ for all $t \in R$ and $x, y \in D^{n \times n}$. Set $u=1 \phi^{-1}$ and $v=1 \phi$. Then

$$
u v=(1 u v) \phi \phi^{-1}=(1 u \phi v) \phi^{-1}=v \phi^{-1}=1,
$$

so $u \in \mathrm{GL}(n, D)$. Also for $t \in R$ we have

$$
v t=1 \phi t=(1 t) \phi=(t 1) \phi=t \theta v,
$$

so

$$
u^{-1} R u=v R v^{-1}=R_{1} \subseteq F^{n \times n} .
$$

9. Suppose $R$ is simple and $E^{\mathrm{op}} \otimes_{F} D$ is a domain for $E$ the division ring component of $R$. Then $\operatorname{dim}_{F} R$ divides $n^{2}$ and $u^{-1} R u \leq F^{n \times n}$ for some $u \in \mathrm{GL}(n, D)$.

Proof. We have $R=E I$ for some set $I=\left\{e_{i j}\right\}$ of matrix units of $R$, say $m \times m$, centralizing $E$. Then $D^{n \times n}=C I \cong C^{m \times m}$ for $C$ the centralizer of $I$ in $D^{n \times n}$, cf. $[13,6.1 .5$.] Then $n=m k$ for some integer $k$ and

$$
E \leq C=D_{1} J \cong D^{k \times k}
$$

for some set $J$ of $k \times k$ matrix units centralizing the copy $D_{1} \supseteq F$ of $D$ in $C$. Apply 8. above to $E$ and $C$. Thus $\operatorname{dim}_{F} E$ divides $k$ and $v^{-1} E v \subseteq F J$ for some unit $v$ of $C$. Then $v \in \operatorname{GL}(n, D)$ and

$$
v^{-1} R v \subseteq v^{-1} E v I \subseteq F J I .
$$

But $J I$ is a set of $n \times n$ matrix units in $D^{n \times n}$ and as such is conjugate to the standard set. Thus $w^{-1} F J I w=F^{n \times n}$ for some $w \in \operatorname{GL}(n, D)$. Now put $u=v w$. Finally $\operatorname{dim}_{F} R=m^{2} \operatorname{dim}_{F} E$, which divides $m^{2} k$ and hence divides $m^{2} k^{2}=n^{2}$. 
10. Suppose $R$ is semisimple and $E^{\mathrm{op}} \otimes_{F} D$ is a domain for each division ring component $E$ of $R$. Then $\operatorname{dim}_{F} R \leq n^{2}$ and $u^{-1} R u \subseteq F^{n \times n}$ for some $u \in \mathrm{GL}(n, D)$. Moreover $\operatorname{dim}_{F} R \leq n$ whenever $R$ is commutative.

Proof. Let $e_{1}, e_{2}, \ldots, e_{s}$ be the primitive central idempotents of $R$, so

$$
R=R e_{1} \times R e_{2} \times \cdots \times R e_{s},
$$

where each $R e_{i}$ is simple. Set $V=D^{(n)}$, row $n$-space over $D$, and regard it as a $D-D^{n \times n}$ bimodule in the obvious way. Then $V=\oplus V e_{i}$ as $D-R$ bimodule. Regard $R e_{i}$ as a subalgebra of $\operatorname{End}_{D} V e_{i}$ and apply 9. Thus if we choose a left $D$-basis $B_{i}$ of $R e_{i}$ there exists $x_{i} \in$ Aut $_{D} V e_{i}$ such that

$$
B_{i} x_{i}^{-1} \operatorname{Re}_{i} x_{i} \subseteq F B_{i}=\bigoplus_{b \in B_{i}} F b .
$$

Then $B=\bigcup B_{i}$ is a left $D$-basis of $V$ and

$$
x=\sum x_{i} \in \bigoplus \operatorname{End}_{D} V e_{i} \subseteq \operatorname{End}_{D} V
$$

is such that $x \in \mathrm{Aut}_{D} V$ and $B x^{-1} R x \subseteq F B$.

Choose $u \in \mathrm{GL}(n, D)$ so that $x^{-1} u$ maps $B$ to the standard basis. Then $u^{-1} R u \subseteq F^{n \times n}$. Also $\operatorname{dim}_{F} R e_{i} \leq\left(\operatorname{dim}_{D} V e_{i}\right)^{2}$ for each $i$ by 9 . again and so

$$
\operatorname{dim}_{F} R=\sum \operatorname{dim}_{F} R e_{i} \leq \sum\left(\operatorname{dim}_{D} V e_{i}\right)^{2} \leq n^{2} .
$$

If $R$ is commutative then each $R e_{i}$ is a field and we can apply 8 . instead of 9 . and obtain $\operatorname{dim}_{F} R e_{i} \leq \operatorname{dim}_{F} V e_{i}$ for each $i$. It then follows here that $\operatorname{dim}_{F} R \leq n$.

11. The Proofs of (1a), 2 and (3a). Now (1a) and 2. follow at once from 8., 9. and 10. Therefore assume the hypotheses and notation of (3a). If $R$ is commutative then $R$ is a direct product of a finite number of fields, each of which is a finite separable extension of $F$. Then $\operatorname{dim}_{F} R \leq n$ and $u^{-1} R u \leq F^{n \times n}$ for some $u \in \mathrm{GL}(n, D)$ by 10 . Moreover if $R$ is a field then $\operatorname{dim}_{F} R$ divides $n$ by 8 .

Now suppose $R$ is a division ring. Then the centre $Z$ of $R$ is separable over $F$. Also $R$ has a strictly maximal subfield $E$ that is separable over $Z$, see [14, p. 245, Proposition]. Then $E$ is separable over $F$ and by the commutative case $\operatorname{dim}_{F} E$ divides $n$. Therefore

$$
\operatorname{dim}_{F} R=\operatorname{dim}_{F} Z\left(\operatorname{dim}_{Z} E\right)^{2}=\left(\operatorname{dim}_{F} E\right)^{2} / \operatorname{dim}_{F} Z
$$

divides $n^{2}$.

Assume now that $R$ is just simple. Then $R=E I$ for some separable division algebra $E$ and set $I$ of matrix units of $R$, say $m \times m$, centralizing $E$. Let $C$ denote the centralizer of $I$ in $D^{n \times n}$. Then

$$
D^{n \times n}=C I \cong C^{m \times m} \text { and } E \leq C \cong D^{k \times k},
$$


where $m k=n$. By the previous case $\operatorname{dim}_{F} E$ divides $k^{2}$. Therefore $\operatorname{dim}_{F} R=$ $m^{2} \operatorname{dim}_{F} E$ divides $m^{2} k^{2}=n^{2}$.

In the general case we obtain $\operatorname{dim}_{F} R \leq n^{2}$ from the case where $R$ is simple exactly as in the proof of 10 .

Finally we have to produce the counterexamples. Let $F, E$ and $A$ be as in the preamble to 7. Then $A \otimes_{F} E \cong E^{2 \times 2}$ and $A$ is isomorphic to a division $F$-subalgebra of $F^{2 \times 2}$. If $A$ is isomorphic to an $F$-subalgebra of $F^{2 \times 2}$ then dimension considerations yield $A \cong E^{2 \times 2}$, which is manifestly false. If $D$ is any purely inseparable field extension of a field $F$ then $D$ satisfies the hypotheses of 3 . by (6a) and clearly there need be no bound on $\operatorname{dim}_{F} D$, or indeed on $\operatorname{dim}_{F} F(x)$ for $x \in D$.

12. Let $n, F$ and $D$ be as in 3. Suppose $Q$ is an $F$-subalgebra of $D^{n \times n}$ with a local system $\mathscr{L}$ of finite-dimensional nilpotent-by-separable $F$-subalgebras. Then $Q=R_{0} \oplus \mathfrak{n}(Q)$, where $R_{0}$ is a finite-dimensional separable $F$-subalgebra of $Q$ and $\mathfrak{n}(Q)$ is the nilpotent radical of $Q$.

Proof. Consider $R, S \in \mathscr{L}$ with $R \leq S$. By a theorem of Wedderburn ([2, p. 386, Theorem 4]) $R=R_{0} \oplus \mathfrak{n}(R)$ for some (separable) $F$-subalgebra $R_{0}$ of $R$. Similarly $S=S_{0} \oplus \mathfrak{n}(S)$. Now $R \cap \mathfrak{n}(S) \leq \mathfrak{n}(R)$, so

(*)

$$
\operatorname{dim}_{F} R_{0}=\operatorname{dim}_{F}(R / \mathfrak{n}(R)) \leq \operatorname{dim}_{F}(R / R \cap \mathfrak{n}(S)) \leq \operatorname{dim}_{F}(S / \mathfrak{n}(S))=\operatorname{dim}_{F} S_{0}
$$

By (3a) we have $\operatorname{dim}_{F} R_{0} \leq n^{2}$. Pick $R \in \mathscr{L}$ so that $\operatorname{dim}_{F} R_{0}$ is maximal. Then the integers in $(*)$ are all equal, $R \cap \mathfrak{n}(S)=\mathfrak{n}(R)$ and $S=R_{0} \oplus \mathfrak{n}(S)$.

If also $T \in \mathscr{L}$ with $S \leq T$ then $S \cap \mathfrak{n}(T)=\mathfrak{n}(S)$ and $T=R_{0} \oplus \mathfrak{n}(T)$.

Consequently $\mathfrak{n}(Q)=\bigcup_{S} \mathfrak{n}(S)$, the union being over all $S \in \mathscr{L}$ with $R \leq S$. Therefore

$$
Q=\bigcup_{S} S=\bigcup_{S}\left(R_{0} \bigoplus \mathfrak{n}(S)\right)=R_{0} \bigoplus \mathfrak{n}(Q),
$$

as required.

13. The Proof of (3b). If $H$ is any finite subgroup of $G$ then $F[H] \leq Q$ is nilpotent-by-separable, e.g. $[4,7.10$, p. 147]. Thus $Q$ satisfies the hypothesis of 12. and hence $Q=R_{0} \oplus \mathfrak{n}(Q)$ as required by (3b).

Let $F, E$ and $A$ be as in the preamble to 7 . with $F$ a subfield of $\mathbf{R}$ and $a=b=-1$. Then $A \otimes_{F} E \cong E^{2 \times 2}$ and $\operatorname{GL}(2, E)$ contains a quaternion subgroup $G$ of order 8 . Since $G$ is finite and $\operatorname{char} F=0$, the unipotent radical of $G$ is trivial. Finally $\operatorname{GL}(2, \mathbf{R})$ contains no copy of $G$ and hence neither does $\mathrm{GL}(2, F)$.

14. The Proof of (1b). We have $F[G]=Q=R_{0} \oplus \mathfrak{n}(Q)$ as in 3b). Since $G$ is locally finite we have $u(G)=G \cap(1+\mathfrak{n}(Q))$, e.g. [16, 1.3.4], so $G / u(G)$ is isomorphic to the image $G_{0}$ of $G$ under the natural projection of $Q$ onto 
$R_{0}$. By (1a) there exists $u \in \mathrm{GL}(n, D)$ with $u^{-1} G_{0} u \subseteq u^{-1} R_{0} u \leq F^{n \times n}$. Consequently

$$
G / u(G) \cong u^{-1} G_{0} u \subseteq \mathrm{GL}(n, F) .
$$

If $G$ has no nontrivial element of order char $F$ then Maschke's theorem and $[16,1.1 .10]$ yield that $Q$ is semisimple, so $Q=R_{0}, G=G_{0}$ and $u^{-1} G u \subseteq$ $\mathrm{GL}(n, F)$.

15. The proof of 4 . If $R$ is at least semisimple the conclusions of 4 . can be proved in the same way as the corresponding conclusions of (3a), see Paragraph 11 . We concentrate on the general case.

Pick a finite field extension $E$ of $F$ such that for $R^{E}=E \otimes_{F} R$ we have $\operatorname{dim}_{E}\left(R^{E} / \mathfrak{n}\left(R^{E}\right)\right)$ minimal. Let $K$ be any finite field extension of $E$. Then after the obvious identifications $R^{K}=\left(R^{E}\right)^{K}$ and $\left(\mathfrak{n}\left(R^{E}\right)\right)^{K} \subseteq \mathfrak{n}\left(R^{K}\right)$. Clearly

$$
\operatorname{dim}_{K}\left(R^{K} /\left(\mathfrak{n}\left(R^{E}\right)\right)^{K}=\operatorname{dim}_{E}\left(R^{E} / \mathfrak{n}\left(R^{E}\right)\right) .\right.
$$

Thus by the choice of $E$ we have $\left(\mathfrak{n}\left(R^{E}\right)\right)^{K}=\mathfrak{n}\left(R^{K}\right)$. But then

$$
\left(R^{E} / \mathfrak{n}\left(R^{E}\right)\right)^{K} \cong R^{K} / \mathfrak{n}\left(R^{K}\right),
$$

which is semisimple. Therefore $R^{E} / \mathfrak{n}\left(R^{E}\right)$ is a separable $E$-algebra, see [14, p. 189, Corollary].

By Wedderburn's theorem again we have $R^{E}=S \oplus \mathfrak{n}\left(R^{E}\right)$ for some separable $E$-subalgebra $S$ of $R^{E}$, so by (3a), or by the opening paragraph of the present proof if you prefer, we have $e=\operatorname{dim}_{E} S \leq n^{2}$ in general and $e \leq n$ if $R$ and hence $S$ is commutative. We are using here that

$$
S \subseteq R^{E} \leq E \bigotimes_{F} D^{n \times n} \cong\left(E \bigotimes_{F} D\right)^{n \times n},
$$

where $E \otimes_{F} D$ is a division $E$-algebra such that $\left.K \otimes_{E} E \otimes_{F} D\right) \cong K \otimes_{F} D$ is a domain for every finite field extension $K$ of $E$.

Let $x_{1}, \ldots, x_{d}$ generate $R$ as an $F$-algebra. Then they generate $R^{E}$ as an $E$-algebra. Let $x_{i}=s_{i}+y_{i}$ where the $s_{i} \in S$ and the $y_{i} \in \mathfrak{n}\left(R^{E}\right)$, and set $Y=\left\{y_{1}, \ldots, y_{d}\right\}$. Pick any $E$-basis $B$ of $S$ and note that $|B|=e$. Now $\mathfrak{n}\left(R^{E}\right)$ is nilpotent with its $n$th power zero, since it embeds into the matrix ring $\left(D^{E}\right)^{n \times n}$. Therefore $R^{E}$ is spanned over $E$ by the set of all elements of the form

$$
b_{0} z_{1} b_{1} z_{2} \cdots z_{m} b_{m}, \quad \text { where the } b_{i} \in B \text {, the } z_{i} \in Y \text { and } 0 \leq m<n \text {. }
$$

If $R$ is commutative then $R^{E}$ is spanned over $E$ by all elements of the form

$$
b_{0} z_{1} z_{2} \cdots z_{m}, \quad \text { where } b_{0} \in B \text {, the } z_{i} \in Y \text { and } 0 \leq m<n \text {. }
$$

Thus $\operatorname{dim}_{F} R=\operatorname{dim}_{E} R^{E} \leq \sum_{i=0}^{n-1} d^{i} e^{i+1}$ in general. In a polynomial ring in $d$ indeterminates, the number of distinct monomials of weight $m \geq 0$ is the binomial coefficient $\left(\begin{array}{c}d+m-1 \\ m\end{array}\right)$. Hence if $R$ is commutative we have

$$
\operatorname{dim}_{F} R \leq \sum_{i=0}^{n-1} e\left(\begin{array}{c}
d+i-1 \\
i
\end{array}\right)
$$


Finally for the counterexample take the same example as used for the corresponding part of $(3 a)$.

\section{ACKNOWLEDGMENT}

The authors would like to express their gratitude to the referee for his useful remarks.

\section{REFERENCES}

1. P. M. Cohn, On the embedding of rings in skew fields, Proc. London Math.Soc. (3) 11 (1961), 511-530.

2. __ Algebra, II, Wiley and Sons, London, 1977.

3. P. M. Cohn and W. Dicks, On central extensions of skew fields, J. Algebra 63 (1980), 143-151.

4. C. W. Curtis and I. Reiner, Methods of representation theory. I, Wiley and Sons, New York, 1981.

5. A. Eisenbud and A. I. Lichtman, On embeddings of group rings of residually torsion-free nilpotent groups into skew fields, Trans. Amer. Math. Soc. 299 (1987), 373-386.

6. N. Jacobson, Structure of rings, A.M.S. Colloq. Publ. vol. 37, Amer. Math. Soc., Providence, R.I. 1956.

7. __, Lectures in abstract algebra. III, Theory of fields and Galois theory, Springer-Verlag, New York, 1961.

8. P. H. Kropholler, P. A. Linnell and J. A Moody, Applications of a new $K$-theoretic theorem to soluble group rings, Proc. Amer. Math. Soc., 104 (1988), 675-684.

9. J. Lewin, Fields of fractions for group algebras of free groups, Trans. Amer. Math. Soc. 190 (1974), 339-346.

10. A. I. Lichtman, On linear groups over a field of fractions of a polycyclic group ring, Israel Math. J. 42 (1982), 318-326.

11. __ Matrix rings and linear groups over a field of fractions of enveloping algebras and groups rings I \& II, J. Algebra 88 (1984) 1-37 and 90 (1984), 516-527.

12. __, Growth in enveloping algebras, Israel J. Math. 47 (1984), 296-304.

13. D. S. Passman, The algebraic structure of group rings, Wiley \& Sons, New York, 1972.

14. R. S. Pierce, Associative algebras, Springer-Verlag, New York, 1982.

15. A. H. Schofield, Representations of rings over skew fields, Cambridge Univ. Press, Cambridge, 1985.

16. M. Shirvani and B. A. F. Wehrfritz, Skew linear groups, Cambridge Univ. Press, Cambridge 1986. 53141

Department of Mathematics, University of Wisconsin-Parkside, Kenosha, Wisconsin

Department of Mathematics, Queen Mary College, London,E1 4NS, United Kingdom 